\title{
Operational models for epidemics of problematic drug use: the Mover-Stayer approach to heterogeneity
}

\author{
Carla Rossi* \\ Department of Mathematics, University of Rome, Tor Vergata, Via Ricerca Scientifica 1, 00133 Rome, Italy
}

\begin{abstract}
A modified version of a susceptibles-infectives-removed (S-I-R) model for the HIV/AIDS epidemic, proposed at the beginning of the 1990s and recently generalized, is presented to mirror the epidemics of problematic drug use. The generalized model, which belongs to the susceptibles-infectives-susceptibles (S-I-S) class of epidemic models, can be used both to estimate interesting epidemic macro-parameters and to make scenario analyses. The model is a compartmental Mover-Stayer-type structure (Soc. Methods Res. 11 (1983) 345; Bull. Narcotics LIII (1-2) (2001) 39; Biometrics 55(4) (1999) 1252; Math. Biosci. 107 (1991) 521 ) and allows taking into account possible heterogeneous behaviours of the susceptibles. Such models, in fact, consider the susceptible population as subdivided into two main groups: the group of stayers, that is, the group of individuals who are considered not at risk of "infection", and the group of movers (possibly divided into sub-groups with different risk behaviour) who are at risk of infection. Due to the interactions between infectious individuals and the susceptibles, some of these may pass to the drug user compartments and begin a "drug user career". The model is presented and studied from a qualitative point of view using a Markov hybrid approximation. Some qualitative evaluation of the possible impact of interventions directed both towards susceptibles (primary prevention) and towards users (secondary prevention and/or law enforcement) are presented. Specific "what if" scenario analyses are obtained by simulation. Possible future developments are outlined.
\end{abstract}

(C) 2003 Elsevier Ltd. All rights reserved.

Keywords: Drug use; Epidemics; Compartmental modelling; Scenario analysis; Evaluation; Drug policy

\section{Introduction: policy needs and dynamic models}

The drug problem and its consequences for society represent a complicated research field. Policy makers and researchers are seeking answers to a number of questions concerning drug use,

\footnotetext{
*Corresponding author. Tel.: + 39-06-72594676; fax: + 39-06-72594699.

E-mail address: rossi@mat.uniroma2.it (C. Rossi).
} 
its consequences, and related costs. They focus on questions such as: "how much drug use?", "what trends are emerging?", "what are the consequences of this use?", "which policies are effective?", "what are the costs of the policies and of the consequences?". The consequences comprise, among many others, the adverse effect of infectious diseases and the costs imposed to society by the drugrelated criminality, and by the burden of disease due to mortality and loss of productivity.

The needs of a policy maker seem remarkably clear if one remains in general terms: it is necessary to describe and understand the problem and follow trends over time, to design proper interventions and to evaluate the results of such interventions. Hence, it is important to understand and "measure" drug use and how it responds to drug control interventions. Mathematical models can contribute to a description and understanding of the various aspects of the problem. They can even help in designing and choosing proper interventions by providing a means of integrating data from different sources, describing a process to increase understanding and simulating policy experiments that are not possible in real life. Important contributions in this direction can be found in [1-3]. The present paper contributes to these objectives by introducing a simple model of drug use, which incorporates various effects on initiation into new use or recidivist use of drugs.

There is evidence that drug use spreads as an infectious disease, i.e. the rate of new cases depends bilinearly on the number of existing cases and on the number of susceptibles [4-6]. Thus, mathematical models developed for epidemiological applications may be of use in this field, although the sociological parameters needed to model drug-related problems may be more transient with respect to the biological parameters used to model infectious disease spread. The length of time that someone remains infectious with a certain disease can be documented, and this infectious period would presumably be the same in different countries; this may be different in the drug field; for example, the reasons for a person ceasing using drugs in London or Amsterdam may be different to those for someone in New York or Sidney and, this may, in turn, result in different values of some characteristic parameters.

\subsection{Compartmental models}

Compartmental models represent a powerful and well-established mathematical tool in modelling the spread of "diseases" in a population. They provide a framework in which numbers of people in different compartments (each one homogeneous with respect to some specified characteristics) and the relationships between such compartments, mirroring the spread of the disease in the population of interest, can be described in mathematical terms.

Two main types of mathematical models have commonly been used to describe the spread of diseases: deterministic models, expressed in terms of systems of differential or difference equations, and stochastic models in terms of stochastic equations or processes. Both types of model assume that the population can be split into compartments. Results from the model usually include the number of people in some compartment of interest, or the number of people moving to and/or from some compartment during a specified time interval.

Once the population has been split into relevant compartments, it is an easy task to describe mathematically how the size of these compartments will change over time by means of suitable equations according to the basic hypotheses of the model describing the dynamics of the population of interest. 
The spread of an epidemic can be modelled either at the micro or macro level. The first approach gives rise to the development of transmission models, the second to the development of operational models. The main difference between the two kinds of models is that transmission models take into account the dynamic processes at the micro level, modelling the interactions between individuals belongings to the different sub-groups involved in the epidemic. Operational models, on the other hand, work on macro-variables, or indicators, suitable to estimating the size of the phenomena or to monitoring the impact of various interventions, as represented by suitable scenario parameters. Many models of the two types have been developed in recent years to study the HIV/AIDS epidemic and are suitable, with some modifications, to model the epidemic of problematic drug use as well.

\subsection{Transmission models}

The complexity of transmission models is due to a detailed formalization of the interactions existing, or supposed to exist, between a large number of subjects involved in the epidemic process. This is the case of the analysis of HIV transmission across risk groups, such as drug users and heterosexuals, when some specific hypothesis on the contact pattern is formulated [7,8], or when the time at which the contact occurs is supposed to somehow affect the probability of transmission of the infection [9]. As already mentioned, such models can be very helpful when specific contact or transmission patterns need to be analysed, but may be extremely cumbersome when the epidemic as a whole is under study. In fact, these detailed model structures contain a variety of parameters regulating every interaction in the diffusion process, while there is seldom sufficient data for robust estimation of such a large number of parameters.

\subsection{Operational models}

A more efficient way of obtaining a simulated epidemic is by using a "simple" operational model. As opposed to transmission models, simple models do not attempt to include all possible group or individual interactions, rather, they summarize the dynamics of the epidemic by some non-linear interaction term and sum up all infected individuals in chains of compartments. In particular, they use the mass-action law in that the number of new infectives is proportional to the product of the number of infectives and the number of susceptibles at risk of infection. Most of the parameters controlling the dynamics in such systems are macro indicators derived from epidemiological studies, external to the model. Their values simply come from monitoring systems, from specialized studies, or from the literature; only a limited number of "internal" parameters is usually left to be estimated by fitting the existing data, or to be used for scenario analyses. Typically, the set of internal parameters includes some form of control of the transmission and of the size of the core group. Other internal parameters may have different origins and interpretations, depending on the design of each model. The ability of correctly describe the epidemic of simple models is theoretically more limited than for complex transmission models. However, in general, they are more tractable and efficient, both because of the limited number of parameters required for their functioning, and because of the robustness of their output. In fact, while the large number of parameters in transmission models often result in their being difficult to identify, parameter estimates in simple models are usually robust enough 
for the model to be successfully used in complex scenario analyses, and under other strained conditions.

Most simple models have a stochastic basis, although their output results in some deterministic forecast curves. Such models, defined as hybrid in [10-12], are the result of the deterministic approximation of stochastic models, using mean times of stay in compartments with conditional Poisson arrivals and maximum likelihood (ML) or numerical estimates of internal parameters. Their use, however theoretically disputable, is more and more frequent among applied researchers because of their technical simplicity and the clarity and immediate possibility of use and interpretation of their results: their output consists of deterministic curves of epidemic indicators (incidence, prevalence, dynamics of the core group, etc.), while the ML estimation of the parameters of interest is derived by the distributional hypotheses of the original stochastic model. One of these models for the HIV/AIDS epidemic was proposed in the early 1990s and has been recently generalized. It allows one to easily obtain scenario analyses $[13,14]$. Such a model, when used jointly with suitable back-calculation methods, allows one to reduce uncertainties in the estimates of incidence curves [15]. A modified version of this model, first presented in [16], is developed and studied herein to mirror the epidemic of problematic drug use. The model can be used both to estimate interesting epidemic macro-parameters and to make scenario analyses. In Section 2, the model is presented. In Section 3, the model is studied from a qualitative point of view to assess the characteristics of the epidemic/endemic behaviour, and to forecast the impact of possible interventions on the spread of the epidemic. Section 4 discusses hypotheses involving the model's parameters. Sections 5 and 6 deal with the simulation procedure and scenario analyses, respectively. Section 7 presents concluding remarks and suggestions for further developments. Mathematical derivations and equations are reported in appendices.

\section{A Mover-Stayer model for the epidemic of problematic drug use}

The graph in Fig. 1 describes the main features of the proposed model: a Mover-Stayer-type structure [17,18] allowing for non-randomness among the susceptibles. According to this model, the susceptible population as subdivided into two groups:

- The Stayers, that is, those individuals who, due to their "prudent" behaviour, cannot be infected and, thus, are not to be at risk. They always remain in the compartment of susceptibles.

- The Movers, who are at risk of infection, represent the so called "core group" [10-12]. They can move to the drug user compartments and begin a "drug user career".

The movers can be infected either by a contact with an infectious individual (drug user) or by a contact with a pusher operating in the black market of drugs.

A drug user passes through a period of "hidden" use at the beginning of his/her career. This period, called "latency period" [19,20], can be split into several different phases. During this period the drug users can:

1. stop using drugs,

2. continue using drugs,

3. die. 


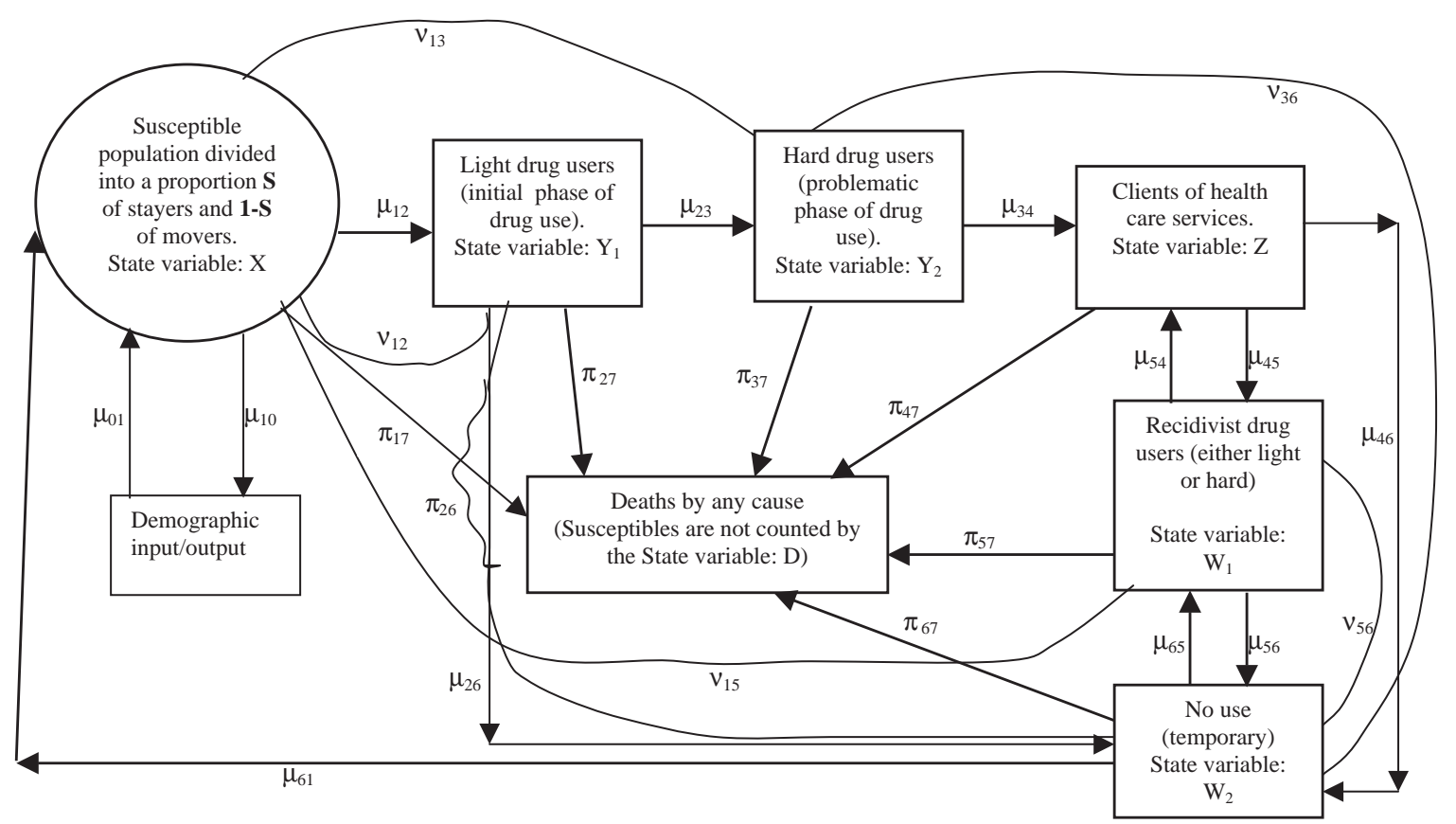

Fig. 1. Compartmental representation of the system dynamic model of the epidemic of problematic drug use.

Afterwards, those who continue using drugs, due to health and criminal problems connected with drug use, are normally recorded by some Agency and becomes "visible". Usually, at this stage, he/she starts to be assisted by health care services and can be cured. However, addictive use of drug is a recidivant syndrome, thus, "recidivist use" is a possible further phase of a drug user career.

The present model uses several compartments to mirror the phenomenon. Similarly to the model proposed in [12], the hidden stage corresponding to the latency period is split into two phases:

- the "light use" phase, which is the initial phase of drug use, wherein light drug users can either stop, die or pass to

- the "hard drug use" phase.

The arrows in the graph of Fig. 1 completely describe all the possible transitions of a drug user career as specified above.

The curves connecting the drug use (infectives) compartments and the susceptible (or temporary no-use) compartments graphically represent the possible interactions between infectious individuals (drug users) and susceptibles. Such interactions, regulated by the mass-action law which produces bi-linear terms in the equations of the model (Appendix A), may cause infections, that is, transitions from susceptibles (or temporary no-use) to infectives. The other possible transitions from susceptibles (or temporary no-use) to infectives are induced by the action of pushers working in the black market and are represented by linear terms in the equations.

In order to write the equations (either deterministic or stochastic), it is necessary to assume further hypotheses and describe the known and unknown parameters. A (possibly marked) 
Markov model is suitable to give a first qualitative insight in the epidemic process. In such a case the length of stay in each compartment is assumed to be exponentially distributed. This implies constant transitions parameters. A more realistic approximation would be based on semi-Markov processes. In such a case the length of stay in each compartment may be assumed to be distributed differently from an exponential. The analysis is more complex but suitable mathematical and simulation techniques can be used. In the present paper, the Markov model is developed and used to make some scenario analyses.

From the graph reported in Fig. 1, the equations of the hybrid Markov model can be easily derived, either in the form of deterministic (continuous or discrete) equations or in the form of stochastic (continuous or discrete) equations. In Appendix A, only the discrete deterministic system of equations is reported, which is the basis of both, the qualitative study of the model, and the simulation procedure. The state variables (other than $S(t)$ which is a proportion) represent the prevalence per million inhabitants, the unit time for numerically integrating the discrete equations is denoted by $D t$. In the simulation runs $D t$ is taken equal to 1 week. The state variables are shortly described in Appendix B.

The equation for the proportion of stayers $S(t)$ is derived under the hypothesis that the new susceptibles, entering in the population of interest, are divided into Stayers and Movers according to constant proportions $S_{0}$ and $M_{0}=1-S_{0}$ (stationarity), with $0<S_{0}<1$ [13]. As a matter of fact, it is reasonable supposing that the adverse consequences of drug abuse, once known, may increase the proportion $S_{0}$. Taking into account this possibility (modification of $S_{0}$ ) would cause higher complexity of the model, thus it is preferable modelling this situation by modulating the proportion of stayers at time $t$, according to the effect of some prevention intervention, measured by an impact parameter $\Delta$, as shown in Section 4 .

In the following section, the qualitative study of the model is considered and the prior evaluation of the efficacy of primary prevention and of secondary prevention and law enforcement is performed. Primary prevention interventions mostly refer to advertising campaigns directed towards susceptibles, whereas secondary prevention interventions essentially comprise campaigns directed towards drug users and harm reduction.

\section{Qualitative study of the epidemic model}

The qualitative study can be performed by jointly analysing the equation for $X$ (susceptibles) and the equation for $S$. The approach is similar to that used in [13]. The analysis focus on the onset incidence indicator, that is, the number of transitions from susceptibles to light drug users per unit time, which is a crucial indicator for monitoring and evaluating drug policy [19].

\subsection{Epidemiclendemic behaviour}

Let us consider the equations for $X(t)$ and for the ratio $S(t+D t) / S(t)$ :

$$
\begin{aligned}
X(t+D t)= & X(t)\left(1+\mu_{01}-\mu_{10}-\pi_{17}\right)-X(t)[1-S(t)]\left[\mu_{12}+v_{12} Y_{1}(t)\right. \\
& \left.+v_{13} Y_{2}(t)+v_{15} W_{1}(t)\right]+\mu_{61} W_{2}(t),
\end{aligned}
$$




$$
\frac{S(t+D t)}{S(t)}=\frac{X(t)\left(1-\mu_{10}-\pi_{17}\right)}{X(t+D t)}+\frac{S_{0}}{S(t)} \frac{\mu_{01} X(t)+\mu_{61} W_{2}(t)}{X(t+D t)} .
$$

Dividing the first one by $X(t+D t)$ and subtracting the second, we obtain

$$
\begin{aligned}
\rho(t)= & 1-\frac{S(t+D t)}{S(t)}=\left[1-\frac{S_{0}}{S(t)}\right] \frac{\mu_{01} X(t)+\mu_{61} W_{2}(t)}{X(t+D t)}-\frac{X(t)}{X(t+D t)}[1-S(t)] \\
& \times\left[\mu_{12}+v_{12} Y_{1}(t)+v_{13} Y_{2}(t)+v_{15} W_{1}(t)\right] .
\end{aligned}
$$

The qualitative analysis of the epidemic is based on the study of the function $\rho(t)$, which can be defined "epidemic/endemic indicator". The details are reported in Appendix C.

To summarize: if $\rho(t)<0$, then $S(t+D t)>S(t)$, the epidemic is spreading and then going towards the endemic phase, whereas if $\rho(t)>0$ then $S(t+D t)<S(t)$ and the endemic phase is ongoing [9]. These two situations are characterized by the following relationships:

$$
\begin{aligned}
\rho(t)>0 \Leftrightarrow & {\left[1-\frac{S_{0}}{S(t)}\right]\left[\mu_{01} X(t)+\mu_{61} W_{2}(t)\right]>X(t)[1-S(t)]\left[\mu_{12}+v_{12} Y_{1}(t)\right.} \\
& \left.+v_{13} Y_{2}(t)+v_{15} W_{1}(t)\right], \\
\rho(t)<0 \leftrightarrow & {\left[1-\frac{S_{0}}{S(t)}\right]\left[\mu_{01} X(t)+\mu_{61} W_{2}(t)\right]<X(t)[1-S(t)]\left[\mu_{12}+v_{12} Y_{1}(t)\right.} \\
& \left.+v_{13} Y_{2}(t)+v_{15} W_{1}(t)\right],
\end{aligned}
$$

where

$$
\left[1-\frac{S_{0}}{S(t)}\right]\left[\mu_{01} X(t)+\mu_{61} W_{2}(t)\right]
$$

represents the "demographic" contribution to the dynamics and:

$$
X(t)[1-S(t)]\left[\mu_{12}+v_{12} Y_{1}(t)+v_{13} Y_{2}(t)+v_{15} W_{1}(t)\right]
$$

represents the "epidemic" contribution to the dynamics (onset incidence).

Further qualitative analyses of the model can be performed under the hypothesis of possible modifications of the parameters or of the dynamics introduced to mirror particular situations. In the following, the analysis of the effect of possible control interventions is briefly outlined.

\subsection{Prior evaluation of the impact of primary and secondary prevention interventions}

Let us consider a primary prevention intervention with efficacy parameter $\Delta$, where $\Delta$ is the probability that a mover becomes a stayer due to the intervention, and let us suppose that both, the intervention and the effect, are observed in the same time unit. In order to evaluate the qualitative impact of the intervention at population level we use the onset incidence indicator [19] as an overall measure. Let us consider the following equations:

$$
\begin{aligned}
\Delta_{1}(t)= & X(t+D t)-X(t)=X(t)\left(\mu_{01}-\mu_{10}-\pi_{17}\right)-X(t)[1-S(t)]\left[\mu_{12}+v_{12} Y_{1}(t)\right. \\
& \left.+v_{13} Y_{2}(t)+v_{15} W_{1}(t)\right]+\mu_{61} W_{2}(t)
\end{aligned}
$$


and

$$
\begin{aligned}
\Delta_{2}(t)= & X(t+D t)-X(t)=X(t)\left(\mu_{01}-\mu_{10}-\pi_{17}\right)-X(t)(1-\Delta)[1-S(t)]\left[\mu_{12}+v_{12} Y_{1}(t)\right. \\
& \left.+v_{13} Y_{2}(t)+v_{15} W_{1}(t)\right]+\mu_{61} W_{2}(t),
\end{aligned}
$$

where the second relationship takes into account the effect of the primary prevention intervention with efficacy parameter $\Delta$. By calculating the difference of the two expressions, we obtain

$$
\begin{aligned}
\Delta_{2}(t)-\Delta_{1}(t) & =X(t) \Delta[1-S(t)]\left[\mu_{12}+v_{12} Y_{1}(t)+v_{13} Y_{2}(t)+v_{15} W_{1}(t)\right] \\
& =X(t) \Delta M(t)\left[\mu_{12}+\xi(t)\right]
\end{aligned}
$$

where

$$
M(t)=1-S(t)
$$

and

$$
\xi(t)=v_{12} Y_{1}(t)+v_{13} Y_{2}(t)+v_{15} W_{1}(t) .
$$

Thus, it is easily seen that the impact of a primary prevention intervention, with efficacy parameter $\Delta$, is proportional to $M(t)$, the proportion of movers among susceptibles, which is monotonically decreasing during the epidemic phase. This implies that the effect of a primary prevention intervention is higher at the beginning of the epidemic. It also implies that the effect of the observation of the adverse consequences of drug abuse cannot be by itself highly effective as primary prevention, due to the long latency period [19]. This prevents from observing such consequences for several years since the beginning of the epidemic. When starting observing them, most movers are already drug users.

Similarly, the effect on initiation of law enforcement interventions can be evaluated by reducing $\mu_{12}$, and the impact of secondary prevention interventions by reducing the $v$ parameters or by modifying the characteristic therapy parameters. A reduction of the sizes of the compartment $Y_{1}$, $Y_{2}$ and $W_{1}$ follows. Thus, the impact of such interventions is more effective during a mature phase of the epidemic when the sizes of the drug use compartments are high.

Thus, $M(t)$ can be used to measure the maximum expected instantaneous impact of primary prevention interventions and $\xi(t)$ can be used to measure the maximum expected instantaneous impact of secondary prevention interventions and law enforcement.

Further qualitative and quantitative analyses are shown in the following scenario section and are obtained by simulation.

\section{The parameters to be externally estimated and the scenario parameters}

In order to perform scenario analyses, we refer to the heroin (by injecting) epidemic which took place in Italy in the past 20 years (beginning estimated around 1980, epidemic peak in 1990-91). Regarding this epidemic, incidence estimates have been obtained by means of back-calculation in the framework of several EU projects [20] and some preliminary results have been published [19]. As a by-product, most parameter values, needed to implement the present model, are available from these studies and from other national epidemiological studies. Thus, only few parameters are used as scenario parameters for the simulation runs in order to obtain "what if" scenarios. These 
are useful for evaluating the impact of possible future epidemics and of control interventions, at least from a qualitative point of view.

For what concerns the parameters needed to implement the simulation program:

- The demographic parameters regulating the dynamics of the susceptible population, namely $\mu_{01}, \mu_{10}$ and $\pi_{17}$, are known and are country-specific.

- The other $\pi$ parameters can be externally estimated from mortality studies among drug users, which are available for most countries in the EU.

- The parameters $\mu_{23}$ and $\mu_{34}$ (natural history parameters) can be estimated on the basis of data available for the study of the latency period $[19,20]$.

- The parameters $\mu_{45}, \mu_{46}, \mu_{54}, \mu_{56}, \mu_{65}$ and $\mu_{61}$ (therapy parameters) can be obtained, at least their order of magnitude, from therapy data available in most countries.

The values of all these parameters for the heroin epidemic in Italy (order of magnitude) are reported in Table 1 . All the other parameters, namely $\mu_{12}, \mu_{26}, v_{12}, v_{13}, v_{15}, v_{26}, v_{36}$ and $v_{56}$ can be used as scenario parameters. The "stationary proportion of Stayers", $S_{0}$ can be used as a scenario parameter too.

All the transition parameters $\mu$ and $\pi$, represent transmission rates per person of the origin compartment per week and appear in the linear terms of the equations, the $v$ parameters

Table 1

Transmission parameters estimated for Italy. Scenario parameters are in bold character (order of magnitude)

\begin{tabular}{|c|c|c|c|}
\hline Between compartments & $\mu$ & $\pi$ & $v$ \\
\hline $0-1$ & 0.00025 & & \\
\hline 1-0 & 0.00002 & & \\
\hline 1-2 & $10^{-5}-10^{-6}$ & & $10^{-5}-10^{-6}$ \\
\hline 1-3 & & & $10^{-6}-10^{-5}$ \\
\hline $1-5$ & & & $10^{-5}-10^{-6}$ \\
\hline 6-1 & 0.0096 & & \\
\hline $1-7$ & & 0.00023 & \\
\hline 2-3 & 0.009 & & \\
\hline $2-6$ & $0.004-0.0004$ & & $10^{-5}-10^{-6}$ \\
\hline $2-7$ & & {$[0.0002-0.0008]^{\mathrm{a}}$} & \\
\hline $3-4$ & 0.004 & & \\
\hline 3-6 & & & $10^{-6}-10^{-5}$ \\
\hline 3-7 & & {$[0.0002-0.0008]^{\mathrm{a}}$} & \\
\hline 4-5 & {$[0.014-0.018]^{\mathrm{b}}$} & & \\
\hline 4-6 & {$[0.007-0.009]^{\mathrm{b}}$} & & \\
\hline 4-7 & & {$[0.0002-0.0008]^{\mathrm{a}}$} & \\
\hline 5-4 & 0.001 & & \\
\hline $5-6$ & {$[0.05-0.1]^{\mathrm{c}}$} & & $10^{-5}-10^{-6}$ \\
\hline $6-5$ & 0.001 & & \\
\hline $5-7$ & & {$[0.0002-0.0008]^{\mathrm{a}}$} & \\
\hline $6-7$ & & {$[0.0002-0.0008]^{\mathrm{a}}$} & \\
\hline
\end{tabular}

${ }^{\text {a }}$ Estimates from mortality studies used within the Consensus Conference on AIDS (Italy, 1998), reported in [14].

${ }^{\mathrm{b}}$ Average length of treatment (hypothesis): $27-36$ weeks.

${ }^{\mathrm{c}}$ Average length of stay in compartment 5 (hypothesis): 5-10 weeks. 
(interaction parameters) are rates per week per pair and appear in the bilinear terms of the equations.

Using a suitable simulation procedure, some impact or sensitivity analysis can be conducted to evaluate the influence of the scenario parameters on the spread of the epidemic.

It would also be possible to make some further scenario analyses to evaluate the impact of an improved efficacy of the therapy services, by taking the scenario parameters as fixed and by using the therapy parameters as variable. Similarly, the natural history parameters could be used to make further "what if" scenario analyses.

\section{The simulation procedure}

The simulation procedure, used to obtain the scenario analyses presented below, is written in S-plus 2000 for PC. All the parameters can be modified at the beginning of each run by means of a user friendly interface. The standard output comprises tables and graphs of the prevalence and incidence curves, related to the various compartments, and of the function $S(t)$ and the various macro-indicators of interest such as $M(t), \rho(t)$ and $\xi(t)$. The total simulation time, which is measured in weeks, can be modified at the beginning of each run.

\section{Some scenario analyses}

Some scenario analyses have been performed to assess the impact of $S_{0}$ and some other parameters on the qualitative and quantitative behaviour of the epidemic. As expected from the results already obtained for the analogous model for the HIV/AIDS epidemic [14], the effect of $S_{0}$ is higher with respect to the other parameters used for the simulation runs.

Some results are reported only in graphic form (Graphs 1-4).

In particular, the graphs presented below show the curves corresponding to: the behaviour of the maximum expected instantaneous impact of primary prevention interventions, measured by $M(t)$, the behaviour of secondary prevention interventions, measured by $\xi(t)$, the behaviour of the epidemic/endemic indicator $\rho(t)$ and the behaviour of the function $S(t)$ (proportion of Stayers). A summary table (Table 2) describes the macro-characteristics of the different simulated epidemics using the locations and the sizes of the peaks of the incidence and prevalence curves, the cumulative incidence of people who ever use drugs and of those who ever progress to heavy use, the value of the cumulative curve of deaths at the end of the period taken into account, and the location of the maximum of the proportion of stayers, which represents the time when the endemic phase starts (saturation effect).

All the values related to incidence and prevalence curves are normalized and expressed per million inhabitants.

\subsection{Basic scenarios}

The basic scenarios are obtained using the second value of the $v$ parameters shown in the corresponding column of Table 1 . This means that we suppose that the interactions between light 

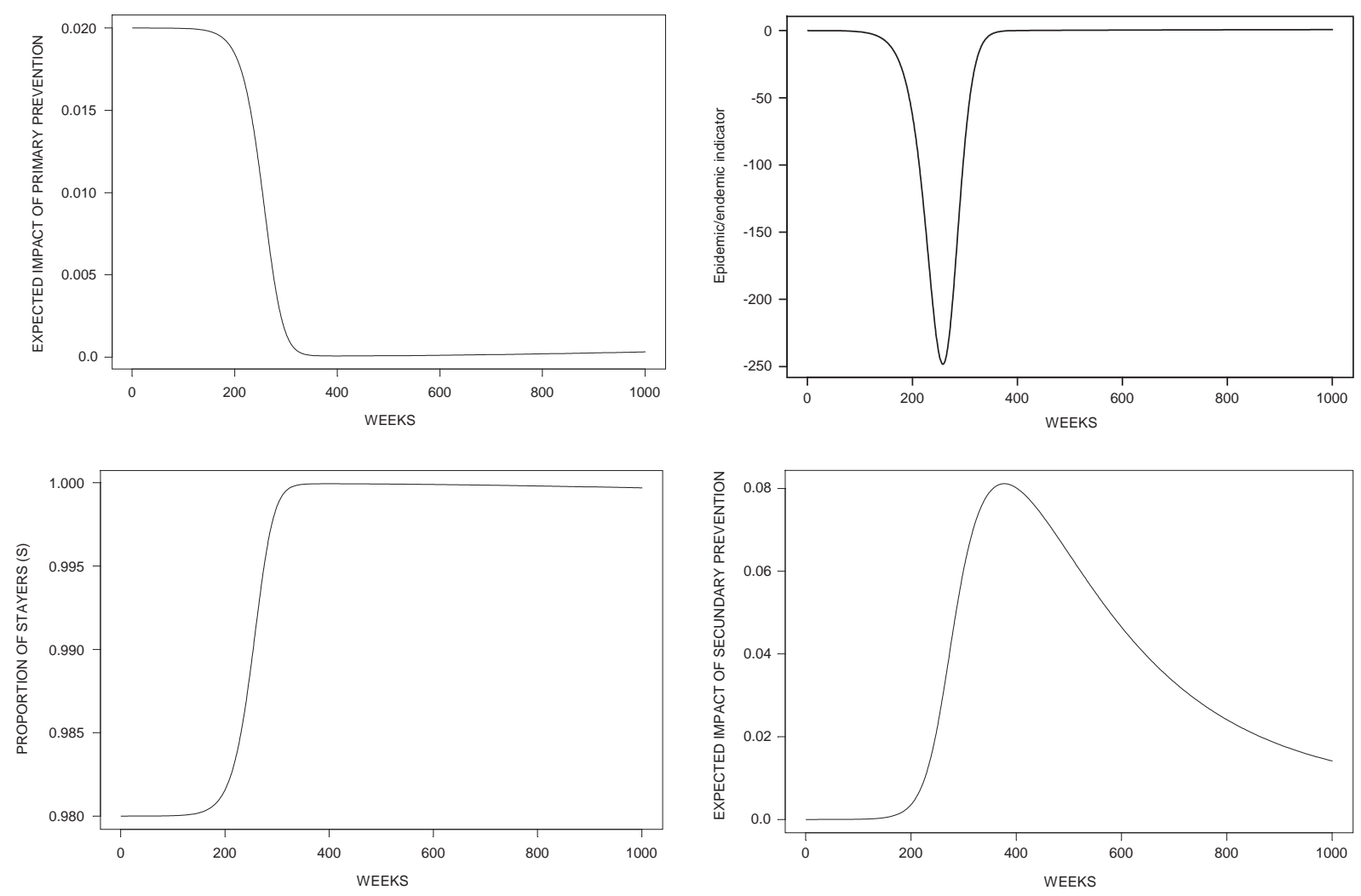

Graph 1. Curve corresponding to the behaviour of the maximum expected effect of a primary prevention intervention $M(t)$, to the behaviour of the epidemic/endemic indicator $\rho(t)$, to the behaviour of the function $S(t)$ (proportion of Stayers) and measuring the maximum expected instantaneous impact of secondary prevention interventions $\xi(t)$ corresponding to basic scenario B1.

drug users and susceptibles, as well as that between recidivist drug users and susceptibles, are less effective than the interactions between hard drug users and susceptibles.

The values of the other parameters used as scenario parameters are reported in the following.

Scenario B1: This scenario is obtained using the parameters reported in Table 1 with $S_{0}=0.98$, $\mu_{12}=10^{-5}, \mu_{26}=0.004$. Various graphs representing the curves of major interest are reported below (Graph 1). Summary statistics are reported in Table 2.

Scenario B2: This scenario is obtained using the parameters reported in Table 1 with $S_{0}=0.99$, $\mu_{12}=10^{-5}, \mu_{26}=0.004$. The graphs representing the curves of interest are reported below (Graph 2). Summary statistics are reported in Table 2.

Scenario B3: This scenario is obtained using the parameters reported in Table 1 with $S_{0}=0.98$, $\mu_{12}=10^{-6}, \mu_{26}=0.004$. Summary statistics are reported in Table 2.

Scenario B4: This scenario is obtained using the parameters reported in Table 1 with $S_{0}=0.98$, $\mu_{12}=10^{-5}, \mu_{26}=0.0004$. Summary statistics are reported in Table 2. 

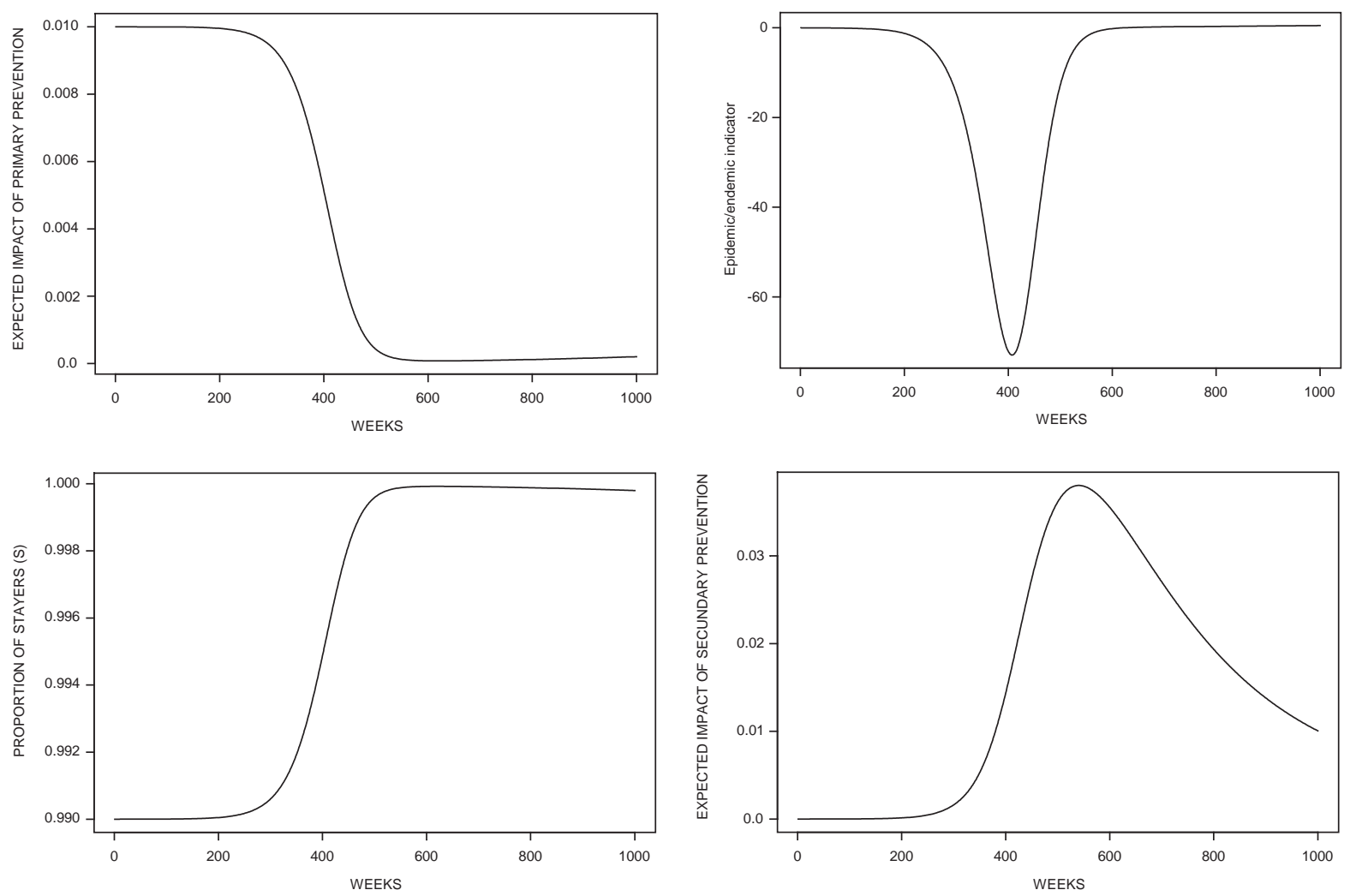

Graph 2. Curve corresponding to the behaviour of the maximum expected effect of a primary prevention intervention $M(t)$, to the behaviour of the epidemic/endemic indicator $\rho(t)$, to the behaviour of the function $S(t)$ (proportion of Stayers) and measuring the maximum expected instantaneous impact of secondary prevention interventions $\xi(t)$ corresponding to basic scenario B2.

\subsection{Alternative v-scenarios}

Scenario A1: This scenario is obtained using the parameters reported in Table 1, but modifying the order of magnitude of the parameters $v\left(10^{-5}\right.$ instead of $10^{-6}$ and viceversa) and with $S_{0}=$ $0.98, \mu_{12}=10^{-5}, \mu_{26}=0.004$. The graphs representing the curves of interest are reported below (Graph 3). Summary statistics are reported in Table 2.

Scenario A2: This scenario is obtained using the parameters reported in Table 1, but modifying the order of magnitude of the parameters $v\left(10^{-5}\right.$ instead of $10^{-6}$ and viceversa) and with $S_{0}=$ $0.99, \mu_{12}=10^{-5}, \mu_{26}=0.004$. The graphs representing the curves of interest are reported below (Graph 4). Summary statistics are reported in Table 2.

By analysing the graphs and the summary statistics reported in Table 2, it is evident that the parameter with the highest impact on the course of the epidemic is $S_{0}$, which is a measure of the size of the group of susceptibles who are at risk of infection (core group). The bigger the core group (the lower $S_{0}$ ) the faster the evolution of the epidemic and the higher the peaks of the prevalence and incidence curves. The impact of the parameter which measures the influence 

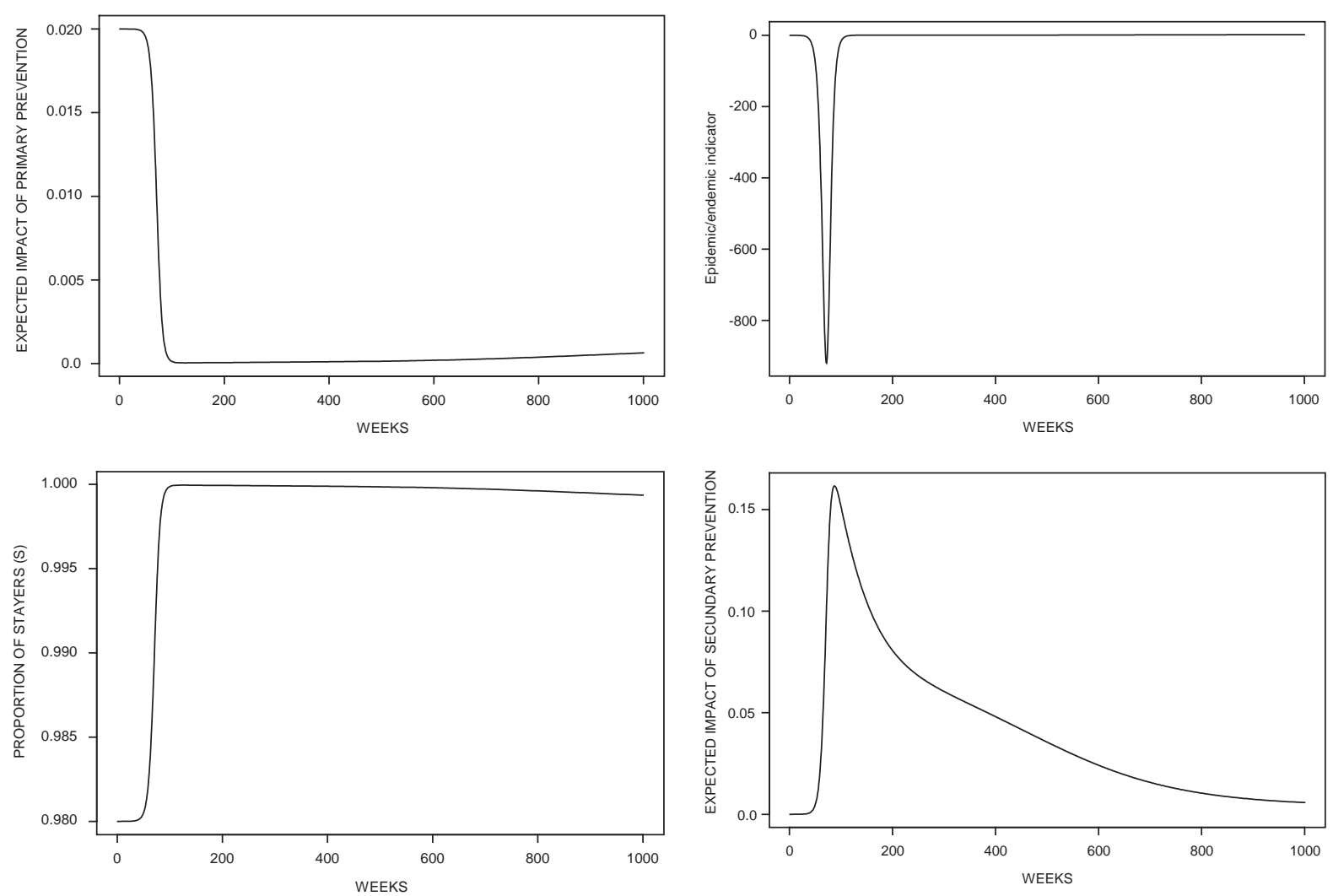

Graph 3. Curve corresponding to the behaviour of the maximum expected effect of a primary prevention intervention $M(t)$, to the behaviour of the epidemic/endemic indicator $\rho(t)$, to the behaviour of the function $S(t)$ (proportion of Stayers) and measuring the maximum expected instantaneous impact of secondary prevention interventions $\xi(t)$ corresponding to alternative $v$ - scenario A1.

of the black market, namely $\mu_{12}$, appears to be lower. Comparing the results obtained under the basic hypothesis on the $v$ parameters, that is, that infectives are mainly hard users, and under the alternative hypothesis, that is, that infectives are mainly light users, it appears that the alternative scenarios show faster evolutions of the epidemics. The qualitative analyses (Section 3) concerning the behaviours of the functions $S(t), \rho(t)$ and of the maximum expected instantaneous impact of primary prevention interventions, measured by $M(t)$, and of secondary prevention interventions, measured by $\xi(t)$, are also confirmed.

It must be stressed again that the stationary proportion of stayers $S_{0}$ is the parameter with the highest impact: there are higher differences in the macro indicators between scenarios 1 and 2 than between scenarios 1 and 3, or between scenarios 2 and 4 .

\section{Conclusive remarks and further developments}

In the present contribution a compartmental model has been developed to reproduce the epidemic of problematic drug use. Both qualitative and quantitative analyses (by means of 

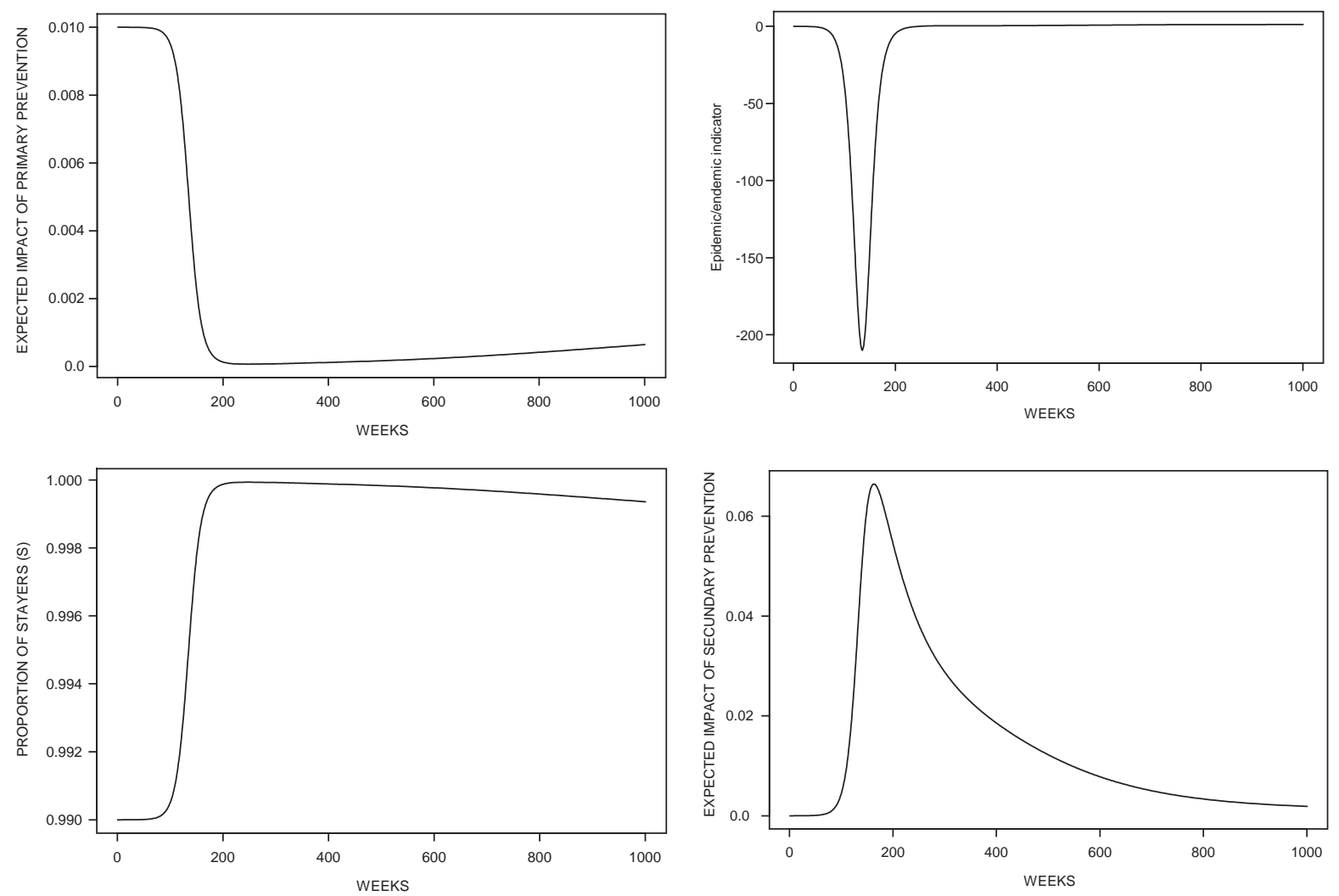

Graph 4. Curve corresponding to the behaviour of the maximum expected effect of a primary prevention intervention $M(t)$, to the behaviour of the epidemic/endemic indicator $\rho(t)$, to the behaviour of the function $S(t)$ (proportion of Stayers) and measuring the maximum expected instantaneous impact of secondary prevention interventions $\xi(t)$ corresponding to alternative $v$-scenario A2.

simulation) have been presented to show the potentiality of the model for decision makers. The quantitative analyses (what if scenarios) have been developed on the basis of the knowledge of the heroin epidemic in Italy in the last 20 years. This allowed to obtain several parameter values from various epidemiological and statistical studies already available. As a matter of fact, heroin by injecting caused the majority of consequences for both health and criminal justice departments in Italy during the 1990s. The majority of treatment requests (about $90 \%$ in the last 10 years) were due to heroin as primary drug abused. Thus, it is clear that the most problematic drug use in Italy still relates to heroin use by injecting. On the other hand, "mutatis mutandis", the results obtained, both from the qualitative and quantitative analyses, apply to other epidemics of drug use, such as new drugs, at least from a qualitative point of view. In particular, the qualitative evaluation of the effectiveness of different types of interventions over the course of the epidemic is valid for any epidemic of problematic use of drugs. As an example one can consider the indications coming from the behaviour of the epidemic/endemic indicator, which changes abruptly in coincidence with changes in the power of policy interventions (notably prevention). This suggests that policy makers should monitor such parameter, which is not directly observable, 
Table 2

Macro-indicators describing the results of the scenario analyses (location is measured in months since the beginning of the epidemic, size of the peak is normalized per million inhabitants)

\begin{tabular}{|c|c|c|c|c|c|c|}
\hline Scenario & $\mathrm{B} 1$ & $\mathrm{~B} 2$ & B3 & B4 & A1 & A2 \\
\hline \multicolumn{7}{|c|}{ Incidence curve from susceptibles to light users } \\
\hline Location of the peak & 50 & 75 & 50 & 50 & 15 & 30 \\
\hline Size of the peak & 1000 & 350 & 1000 & 1000 & 3500 & 850 \\
\hline Cumulative incidence & 20,000 & 14,000 & 20,000 & 20,000 & 35,000 & 18,000 \\
\hline \multicolumn{7}{|c|}{ Prevalence curve of light users } \\
\hline Location of the peak & 62 & 90 & 62 & 62 & 20 & 40 \\
\hline Size of the peak & 10,000 & 3800 & 10,000 & 12,000 & 15,000 & 6200 \\
\hline \multicolumn{7}{|c|}{ Incidence curve from light users to hard users } \\
\hline Location of the peak & 62 & 90 & 62 & 62 & 20 & 40 \\
\hline Size of the peak & 350 & 150 & 350 & 500 & 550 & 250 \\
\hline Cumulative incidence & 16,000 & 6000 & 16,000 & 18,000 & 20,000 & 6000 \\
\hline \multicolumn{7}{|c|}{ Prevalence curve of hard users } \\
\hline Location of the peak & 80 & 125 & 80 & 80 & 50 & 60 \\
\hline Size of the peak & 7500 & 3500 & 7500 & 9000 & 8000 & 3800 \\
\hline \multicolumn{7}{|c|}{ Incidence curve from hard users to therapy } \\
\hline Location of the peak & 90 & 110 & 90 & 90 & 50 & 60 \\
\hline Size of the peak & 120 & 60 & 120 & 150 & 120 & 50 \\
\hline \multicolumn{7}{|c|}{ Prevalence curve of clients of therapy services } \\
\hline Location of the peak & 100 & 125 & 100 & 100 & 62 & 64 \\
\hline Size of the peak & 1200 & 600 & 1200 & 1600 & 1300 & 620 \\
\hline \multicolumn{7}{|c|}{ Prevalence curve of recidivist use } \\
\hline Location of the peak & 100 & 125 & 100 & 100 & 62 & 64 \\
\hline Size of the peak & 2700 & 800 & 2700 & 3000 & 2500 & 700 \\
\hline \multicolumn{7}{|c|}{ Prevalence curve of no use (temporary) } \\
\hline Location of the peak & 130 & 150 & 130 & 150 & 80 & 80 \\
\hline Size of the peak & 3000 & 1500 & 3000 & 2600 & 3200 & 1700 \\
\hline \multicolumn{7}{|l|}{ Cumulative death curve } \\
\hline Final value & 4100 & 1800 & 4100 & 5000 & 4100 & 1800 \\
\hline \multicolumn{7}{|l|}{ Proportion of stayers } \\
\hline Location of the peak & 70 & 100 & 70 & 62 & 18 & 40 \\
\hline
\end{tabular}

using indirect estimates, such as onset incidence estimates, obtained by various estimation methods [20] or survey data. In fact, the epidemic part of the indicator is essentially based on incidence of new use, whereas the demographic part is easily obtained on the basis of census statistics and therapy data. As a consequence, decision makers should organise real-time monitoring systems allowing for onset incidence estimation. 
The use of suitable markers (marked processes) would allow to incorporate further descriptions of each individual involved, for instance a mark might take into account the numbers of incarcerations, or the numbers of failed therapy interventions, or the numbers of non-fatal overdoses and so on. In particular, possible repeated therapy interventions should be incorporated in order to get a more realistic model of a drug user career. There is a general agreement that the time spent in the therapy compartment for the first therapy episode is differently distributed with respect to those related to the following episodes and this should be taken into account. The complexity of such analysis is evident and the interpretation of the results might be quite uncertain and unreliable, due to the great number of unknown parameters which are presently not available from epidemiological studies. Thus, it is much better to wait until the therapy data-sets comprise reliable and complete information to allow the estimation of the parameters of interest for scenario analyses.

In order to obtain a more realistic model, the transition parameters should not be taken as constant but should be represented as functions taking into account the history of drug use for any individual, represented by statistical variables supposed known (covariates), and the history of policy interventions (availability of services, law enforcement activities), represented by other covariates (time dependent) and, possibly, by latent variables. This would result in a very realistic but, at the same time, unreliable and intractable transmission micro-model of no use.

Further generalizations might concern a more realistic approach to modelling the length of stay in the various compartments, taking into account the heterogeneity of individual behaviours as resulting from studies on latency period [19,20]. This issue will be addressed in a future contribution by means of a proper simulation model.

\section{Acknowledgements}

This work has been partially founded by CNR, progetto strategico "Metodi e modelli matematici nello studio dei fenomeni biologici". The work benefited from the EU funded 'European Network to develop Policy Relevant Models and Socio-Economic Analyses of Drug Use, Consequences and Interventions' (TSER/DGXII project ERB 4141 PL980030).

\section{Appendix A. Deterministic difference equations of the model}

(1) Susceptible population: state variable: $X$

$$
\begin{aligned}
X(t+D t)= & X(t)\left(1+\mu_{01}-\mu_{10}-\pi_{17}\right)-X(t)[1-S(t)]\left[\mu_{12}+v_{12} Y_{1}(t)\right. \\
& \left.+v_{13} Y_{2}(t)+v_{15} W_{1}(t)\right]+\mu_{61} W_{2}(t) .
\end{aligned}
$$

(2) Light drug users (initial phase of drug use): state variable: $Y_{1}$

$$
\begin{aligned}
Y_{1}(t+D t)= & Y_{1}(t)\left(1-\mu_{23}-\mu_{26}-\pi_{27}\right)+X(t)[1-S(t)]\left[\mu_{12}+v_{12} Y_{1}(t)\right. \\
& \left.+v_{13} Y_{2}(t)+v_{15} W_{1}(t)\right] .
\end{aligned}
$$


(3) Hard drug users (problematic phase of drug use): state variable: $Y_{2}$

$Y_{2}(t+D t)=Y_{2}(t)\left(1-\mu_{34}-\pi_{37}\right)+\mu_{23} Y_{1}(t)$.

(4) Clients of health care services: state variable: $Z$

$Z(t+D t)=Z(t)\left(1-\mu_{45}-\mu_{46}-\pi_{47}\right)+\mu_{34} Y_{3}(t)+\mu_{54} W_{1}(t)$.

(5) Recidivistic drug users: state variable: $W_{1}$

$$
\begin{aligned}
W_{1}(t+D t)= & W_{1}(t)\left(1-\mu_{54}-\mu_{56}-\pi_{57}\right)+W_{2}(t)\left[\mu_{65}+v_{26} Y_{1}(t)\right. \\
& \left.+v_{36} Y_{2}(t)+v_{56} W_{1}(t)\right]+\mu_{45} Z(t) .
\end{aligned}
$$

(6) No use (temporary): state variable: $W_{2}$

$$
\begin{aligned}
W_{2}(t+D t)= & W_{2}(t)\left(1-\mu_{61}-\pi_{67}\right)-W_{2}(t)\left[\mu_{65}+v_{26} Y_{1}(t)+v_{36} Y_{2}(t)+v_{56} W_{1}(t)\right] \\
& +\mu_{26} Y_{1}(t)+\mu_{46} Z(t)+\mu_{56} W_{1}(t) .
\end{aligned}
$$

(7) Deaths from drug user population: state variable: $D$

$D(t+D t)=D(t)+\pi_{27} Y_{1}(t)+\pi_{37} Y_{2}(t)+\pi_{47} Z(t)+\pi_{57} W_{1}(t)+\pi_{67} W_{2}(t)$.

(8) Proportion of stayers: state variable: $S$

$S(t+D t)=S(t) \frac{X(t)\left(1-\mu_{10}-\pi_{17}\right)}{X(t+D t)}+S_{0} \frac{\mu_{01} X(t)+\mu_{61} W_{2}(t)}{X(t+D t)}$.

\section{Appendix B. State variables and parameters of the Markov model}

$\mu_{i j} \quad$ linear transition rates (constant)

$\pi_{i j} \quad$ mortality rates (constant)

$v_{i j} \quad$ interaction rates (constant)

$X(t)$ population of susceptibles (prevalence)

$S(t)$ proportion of stayers

$M(t)$ proportion of movers

$Y_{1}(t)$ light drug users (prevalence)

$Y_{2}(t)$ hard drug users (prevalence)

$Z(t)$ clients of health care services (prevalence)

$W_{1}(t)$ recidivist drug users (prevalence)

$W_{2}(t)$ temporary non-users (prevalence)

$D(t)$ deaths by any cause (cumulative prevalence)

\section{Appendix C. Study of the epidemic/endemic indicator}

Relation (1) implies that if $S(t)=1$ (all the susceptibles are stayers) then the epidemic contribution vanishes, $S(t)$ is decreasing $(\rho(t)>0)$ and the endemic phase starts, whereas, from relation (2), it follows that if $S(t) \leqslant S_{0}$, then $S(t)$ is increasing $(\rho(t)<0)$, thus, there exists a time $t^{*}$ 
such that for $t>t^{*} S_{0} \leqslant S(t) \leqslant 1$. It follows that the values $S_{1}=1$ and $S_{2}=S_{0}$ are reflecting barriers for the stochastic process for $t>t^{*}$; thus, it exists a positive value $\varepsilon\left(0<\varepsilon<1-S_{0}\right)$ such that if $S(t)>1-\varepsilon$, then the endemic phase of the epidemic starts and $S(t)$ becomes a decreasing function, but, as soon as $S(t)<1-\varepsilon$ then $S(t)$ becomes an increasing function producing new smaller epidemic waves such as those observed periodically for any epidemic. In other words, we can say that the relative influence of the epidemic term is increasing for $S(t)$ decreasing, whereas the relative influence of the demographic term is decreasing for $S(t)$ decreasing. We can define the value $S^{*}=1-\varepsilon^{*}$ the threshold epidemic value.

\section{References}

[1] Behrens DA, Caulkins JP, Tragler G, Haunschmied JL, Feichtinger G. A dynamic model of drug initiation: implications for treatment and drug control. Mathematical Biosciences 1999;159:1-20.

[2] Behrens DA, Caulkins JP, Tragler G, Feichtinger G. Optimal control of drug epidemics: prevent and treat-but not at the same time? Management Science 2000;46(3):333-47.

[3] Billard L, Dayananda PWA. Drug addiction-pusher generated from addicts. Biometrical Journal 1993;35:227-44.

[4] De Alarcòn R. The spread of a heroin abuse in a community. Bulletin on Narcotics 1969;21:17-22.

[5] Hunt LG, Chambers CD. The heroin epidemics. New York: Spectrum Publications Inc.; 1976.

[6] Mackintosh DR, Stewart GT. A mathematical model of a heroin epidemic: implications for control policies. Journal of Epidemiology and Community Health 1979;33:299-304.

[7] Hadeler KP. Modeling AIDS in structured populations. In: Proceedings of I.S.I. 47th Session. International Statistical Institute, Paris, 1989. p. 83-99.

[8] Jacquez JA, Simon CP, Koopman J, Sattenspiel L, Perry T. Modeling and analyzing HIV transmission: the effect of contact patterns. Mathematical Biosciences 1988;92:119-99.

[9] Bailey NTJ. A revised assessment of the HIV/AIDS incubation period, assuming a very short early period of high infectivity and using only San Francisco public health data on prevalence and incidence. Statistics in Medicine 1997; 16:2447-58.

[10] Bailey NTJ. The use of operational modelling of HIV/AIDS in a system approach to public health decision making. Mathematical Biosciences 1991;107:413-30.

[11] Bailey NTJ. An improved hybrid HIV/AIDS model geared to specific public health data and decision making. Mathematical Biosciences 1993;117:221-37.

[12] Bailey NTJ. HIV/AIDS Core-group dynamic and public health action. In: Kaplan EH, Brand eau ML, editors. Modeling the AIDS epidemic. New York: Raven Press; 1994. p. 29-52.

[13] Rossi C. A Mover-Stayer type model for problem drug use epidemic. Bulletin on Narcotics 2001;LIII(1-2):39-64.

[14] Rossi C, Schinaia G. The Mover-Stayer model for the HIV/AIDS epidemic in action. Interfaces 1998;28(3): $127-43$.

[15] Ravà L, Rossi C, Pasqualucci C, Schinaia G. Estimating the size of the HIV/AIDS epidemic: complementary use of empirical Bayesian back calculation and the Mover-Stayer model for gathering the largest amount of information. Simulation 1998;71(4):213-27.

[16] Rossi C. A stochastic Mover-Stayer model for HIV epidemic. Mathematical Biosciences 1991;107:521-45.

[17] Albert PS. A Mover-Stayer model for longitudinal marker data. Biometrics 1999;55(4):1252-7.

[18] Morgan TM, Aneshensel CS, Clark VA. Parameter estimation for Mover-Stayer models: analyzing depression over time. Sociological Methods and Research 1983;11:345-66.

[19] Ravà L, Calvani MG, Wiessing L, Heisterkamp S, Rossi C. Incidence indicators for policy making: models, estimation and implications. Bulletin on Narcotics 2001;LIII(1-2):2001.

[20] Rossi C, Wiessing L, et al. Pilot project to estimate time trends and incidence of problem drug use in the European Union, EMCDDA, Lisbon, 1999. 